\title{
Small bowel adenocarcinoma (SBA) - a case report
}

\author{
Joanna Polczyk
}

Backround. Small bowel cancers account for less than $0.5 \%$ of all malignancies and approximately $1-3 \%$ of gastrointestinal tract malignancies. The two major tumour types are carcinoid and adenocarcinoma. The latter is most commonly found in the duodenum and jejunum, but least often in the ileum.

Case presentation. We present a case study of a 67-year-old woman with small bowel adenocarcinoma (SBA) of the ileum. The patient was admitted to the hospital with nonspecific, persistent, recurrent pain in her upper abdomen and with suspected subileus. Previously performed clinical investigation (i.e. colonoscopy, computed tomography scans - CT scans, small bowel follow-through) didn't reveal any abnormalities. At laparotomy we discovered a round mass obstructing the ileal lumen at about $40 \mathrm{~cm}$ from the Bauhin valve. A tumour was then resected within its surgical margins. Pathologic examination revealed an ileal adenocarcinoma and an infiltration of the local adipose tissue (Adenocarcinoma G3 partim gelatinosum), with no evidence of metastatic lesions in the lymph nodes nor into other organs (pT3NOM0; the clinical stage IIA). Further oncological treatment was thereby advised.

Conclusions. A low prevalence and non-specific symptoms along without any clearly established diagnostic nor treatment protocols makes a final diagnosis difficult and thus merits further diagnostic investigation, particularly the testing of specific biochemical or immunological markers.

NOWOTWORY J Oncol 2017; 67, 2: 137-141

Key words: adenocarcinoma, fluoropirymidine, jejunum, KRAS, oxaliplatin, small bowel

\section{Introduction}

The small bowel comprises $75 \%$ of intestinal length and $90 \%$ of the gastrointestinal tract surface [1]. Small bowel malignancies account for less than $0.5 \%$ of all the malignancies and about 1-3\% gastrointestinal tract malignancies [2]. In 2013, small bowel malignancies had been diagnosed in 126 polish women and 134 polish men [3]. The most common histological types of small bowel cancer are adenocarcinoma and carcinoid tumour. The others include lymphoma, sarcoma and gastrointestinal stromal tumours (GIST) (Tab. I), [2]. Small bowel adenocarcinoma (SBA) is most commonly located in the duodenum (57\%) and jejunum (29\%), whereas it is most rarely found in the ileum $(10 \%)[1,4,5]$, while carcinoid tumour and sarcoma are most commonly found in the ileum $[1,2,6]$.

The risk factors for SBA include: familial adenomatous polyposis (FAP), Lynch Il syndrome (HNPCC, hereditary non- -polyposis colorectal cancer), Peutz-Jeghers syndrome, sporadic polyps, Crohn's disease, coeliac disease, AIDS, a history of cholecystectomy, smoking and alcohol consumption $[1,2,5,7]$. The median age at diagnosis for SBA is 67 years with over $85 \%$ of patients presenting after age 50 [1]. Clinical presentation of SBA is asymptomatic in the long term, which leads to diagnosis being delayed by an average of about 6 months [4]. When symptoms successively develop, they present as nonspecific abdominal discomfort and/or: weight loss, anaemia, gastrointestinal bleeding, diarrhea and postprandial abdominal pain. Ultimately, at locally advanced stages, the patient is admitted to hospital with so called 'acute abdomen' symptoms [1, 2, 5, 8].

Standard available diagnostic procedures like abdominal ultrasound, oesophagogastroduodenal endoscopy and colonoscopy enable the diagnosis of only those tumours localised in duodenum, whereas abdominal computed to-

Department of General and Endocrine Surgery, School of Medicine with the Division of Dentistry in Zabrze,

Medical University of Silesia, Katowice, Poland

Department of General Surgery, Municipal Hospital, Zabrze, Poland 
Table I. Incidence of the most common pathological types of small bowel malignancies $[2,4]$

\begin{tabular}{lcccccc}
\hline Pathological type of small bowel malignancies & Carcinoid & Adenocarcinoma & Lymphoma & Sarcoma & GEP-NET & GIST \\
\hline The incidence in \% & $35-42$ & $30-40$ & $15-20$ & $10-15$ & $20-50$ & 7 \\
\hline
\end{tabular}

GIST — gastrointestinal stromal tumour; GEP-NET — gastroenteropancreatic neuroendocrine tumours

mography scans (CT scans) do not usually visualize any abdominal mass but only visualize nonspecific changes in the small bowel like swollen lymph nodes or thickening of the bowel wall. Without any principal diagnosis, with only a suspicion of lesions in the distal segment of small bowel, this then prompts the use of more advanced methods: endoscopy (video capsule endoscopy, double-balloon assisted enteroscopy), imaging techniques using computed tomography (CT) and magnetic resonance; CT/MRI enteroclysis and CT/MRI enterography. Furthermore, arteriography, scintigraphy and small bowel follow-through are increasingly becoming more important for evaluating small bowel disease [1, 2, 5, 7, 8]. Despite the wide spectrum these aforementioned diagnostic methods we are still missing a specific screening method that could simultaneously meet the following criteria: cost, availability, precision of intestinal lumen evaluation together being able to take samples for pathological examination, and invasiveness. Despite these aforementioned procedures being all performed in Poland, they are unfortunately only readily available usually at highly specialized medical centres.

\section{Case presentation}

A 67-year old female patient, 6 months after a previously performed laparoscopic cholecystectomy due to cholecystolithiasis was re-admitted to the Department of General Surgery at the Zabrze Municipal Hospital suspected with an incomplete small bowel obstruction. She had a medical history of hypothyroidism, chronic obturative pulmonary disease, type 2 diabetes mellitus, hypertension and colonic diverticular disease.

For about the last 6 months, the patient experienced recurrent epigastric colicky pain and abdominal distention. After one of the first episodes, the patient underwent a full diagnostic procedure in the aforementioned Department of General Surgery, which did not reveal any abnormalities. An oesophagogastroduodenal endoscopy performed at that time demonstrated bile reflux erosive gastritis. In a colonoscopy, hyperplastic polyps were simultaneously excised in the caecum, near the orifice of the vermiform appendix, transverse colon, sigmoid colon and rectum. Small bowel follow-through with gastrografin also did not reveal any abnormalities (Fig. 1). CT scans of the abdomen and pelvis demonstrated an intestine without increased fluid levels, but with only a segmental jejunal dilatation up to about $29 \mathrm{~mm}$ accompanied with a segmental ileal wall thickening of up to about 11-13 mm diameter suggesting the presence of an inflammatory process. No swollen lymph nodes in the abdomen nor pelvis were found (Fig. 2). Laboratory tests were unremarkable except for slightly elevated inflammation parameters (Tab. II).

The constant upper abdominal pain was suspected to result from post-cholecystectomy syndrome of the bile reflux gastritis. The reason why the patient attended our Department was that this abdominal pain was continuously lasting for several days. She experienced vomiting several times daily before and on admission. She was passing gas regularly and the last defecation had occurred a week before admission to the Department.

Physical examination on admission revealed a soft abdomen with tenderness in the upper middle quadrant. No pathological mass was palpated in the abdomen. Peristalsis was present, with no metallic tinkling, and digital rectal examination showed the rectal ampulla full of brown stool. Plain abdominal radiography demonstrated intestines with increased fluid levels in the left abdomen; there being no free gas within the peritoneal cavity (Fig. 3).

Due to patient's state deteriorating and aggravated abdominal pain, a decision for emergency surgery was made. The abdomen was opened with a midline incision centreing the umbilicus. There was a small amount of serous fluid in the abdominal cavity which was collected for microbiology investigation. The small intestine was dilated with a thickened and overworked wall extending almost the whole length up to the obstacle. About $40 \mathrm{~cm}$ from the Bauhin valve a tumour was found to occlude the bowel lumen and was invading directly into the neighbouring mesentery. A radical resection of the pathologically changed ileum segment and the entire tumour along with its mesenteric involvement was performed and the specimen sent for pathologic investigation. Gastrointestinal tract anastomosis was performed by a side-to-side ileo-ileal anastomosis technique with double-layer continuous sutures. The mesentery of the small intestine was closed with interrupted sutures to prevent internal hernia. After controlling the bleeding, the abdominal cavity was flushed with a warm saline solution. The abdomen was then closed in layers and sterile dressing applied to the wound.

The pathological examination of the resected specimen demonstrated ileal adenocarcinoma (G3 partim gelatinosum) infiltrating local adipose tissue, with all the lymph nodes being negative and no metastases to other organs (pT3NOM0; the clinical stage IIA). The level of carcino-embryonic antigen (CEA) was normal ( $<5 \mathrm{mg} / \mathrm{mL}$ ).

The postoperative period was uncomplicated and the patient was discharged on the 12th postoperative day in 


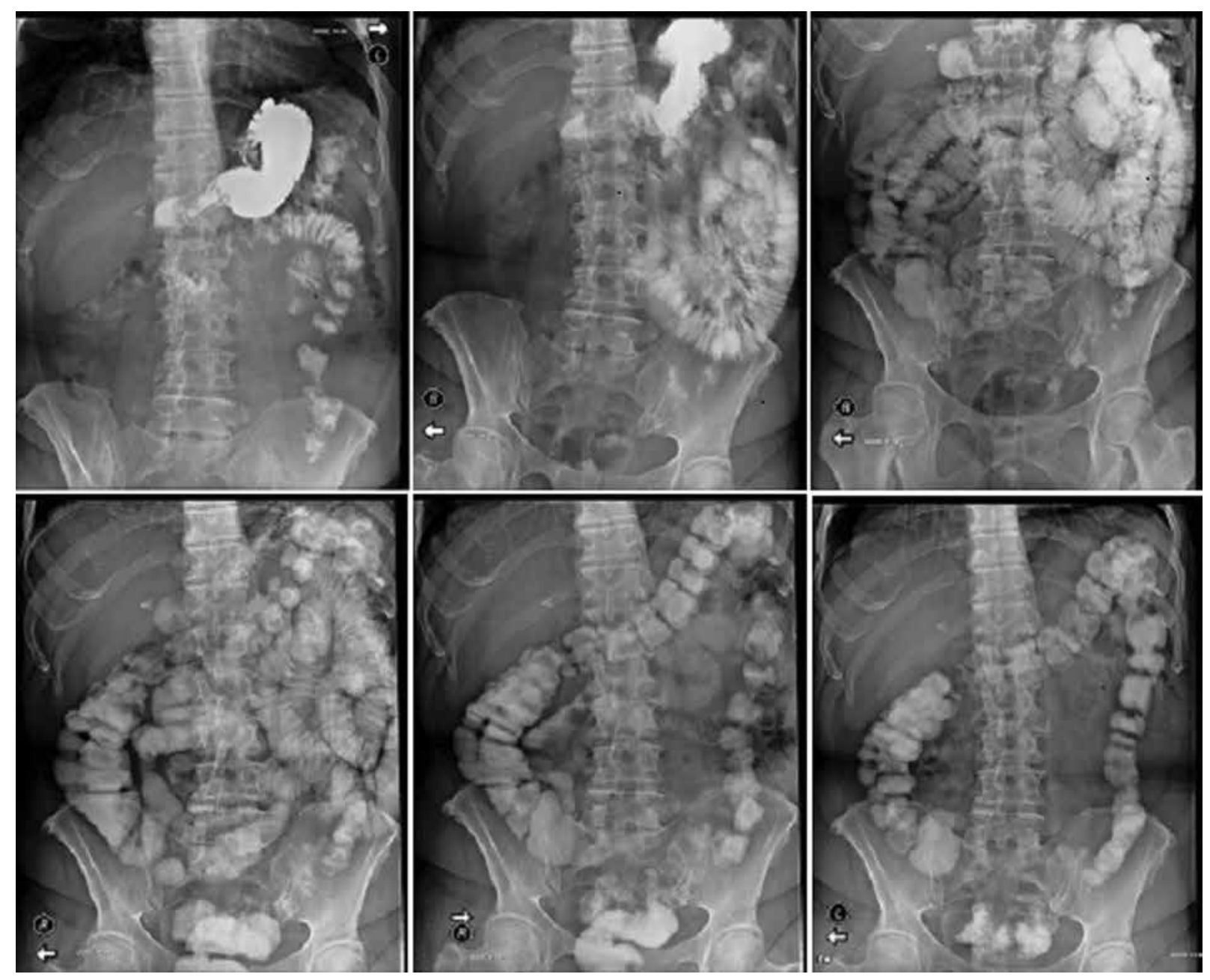

Figure 1. Small bowel follow-through with gastrografin. A sequence of images visualizing the contrast reaching towards the end of the small intestine

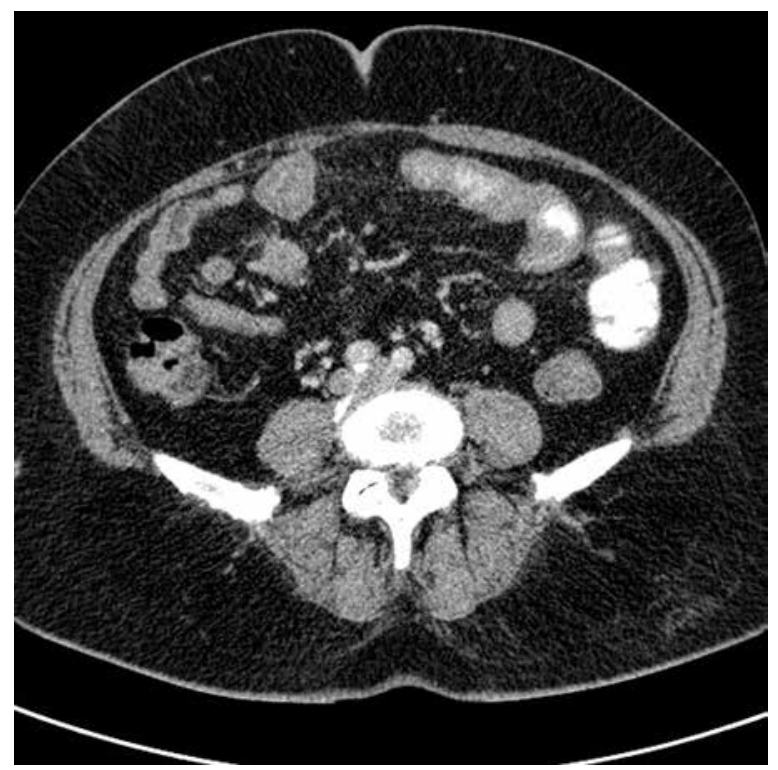

Figure 2. Abdominal and pelvic CT scans with contrast. We observe segmental wall thickening of the ileal loop a satisfactory condition for further systemic treatment at the Department of Radiotherapy and Chemiotherapy in the Centre of Oncology, Maria Skłodowska-Curie Memorial Institute, Gliwice Branch, where she is continuing a fifth cycle of LF1 (5-Fluorouracil+Sodium Levofolinate).

\section{Discussion}

Our case study has shown that an early and accurate diagnosis of small bowel malignancies is still difficult to achieve and is often only possible intraoperatively. Recent improvements in advanced diagnostic imaging methods and developments to video capsule endoscopy has improved the numbers of correct diagnoses made of small bowel malignancies, however this procedure is still barely available in Poland [8]. The lack of there being any unambiguous biochemical or immunological markers together with nonspecific symptoms often delays diagnosis, consequently prolonging the time for delivering adequate oncological treatment. It thus appears necessary to seek for new, easy, highly sensitive and highly specific markers of SBA. 
Table II. Laboratory tests results during hospitalization

\begin{tabular}{|c|c|c|c|c|c|c|c|}
\hline Date/Lab.test & $\begin{array}{c}\text { CRP } \\
{[\mathrm{mg} / \mathrm{L}]}\end{array}$ & 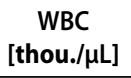 & $\begin{array}{c}\text { RBC } \\
\text { [mill./ } \mu \mathrm{L}]\end{array}$ & $\begin{array}{l}\mathrm{Hgb} \\
{[\mathrm{g} / \mathrm{dL}]}\end{array}$ & $\begin{array}{c}\text { PLT } \\
\text { [thou./} / \mu \mathrm{L}]\end{array}$ & $\begin{array}{c}\text { Creatinine } \\
{[\mathrm{mg} / \mathrm{dl}]}\end{array}$ & $\begin{array}{l}\text { Potassium } \\
\text { [mmol/l] }\end{array}$ \\
\hline 18.04 & 7.39 & 12 & 4.2 & 15 & 340 & 0.92 & 3.9 \\
\hline 22.04 & 13.32 & 15 & 4.65 & 13.8 & 397 & 0.74 & 4 \\
\hline 26.04 & 10.31 & 10.9 & 4.06 & 12.9 & 352 & 0.89 & 4.51 \\
\hline 11.05 & 44 & 10 & 4.51 & 13.5 & 374 & 1.47 & 5.23 \\
\hline 17.05 & 20 & 10.7 & 3.99 & 11.9 & 371 & 0.9 & 3.74 \\
\hline 8.06 & 4.42 & 12.1 & 5.3 & 15.3 & 437 & 1.39 & 4.22 \\
\hline
\end{tabular}

WBC — white blood cell count; RBC — red blood cell count; PLT — platelets; CRP — C reactive protein; Hgb — hemoglobin

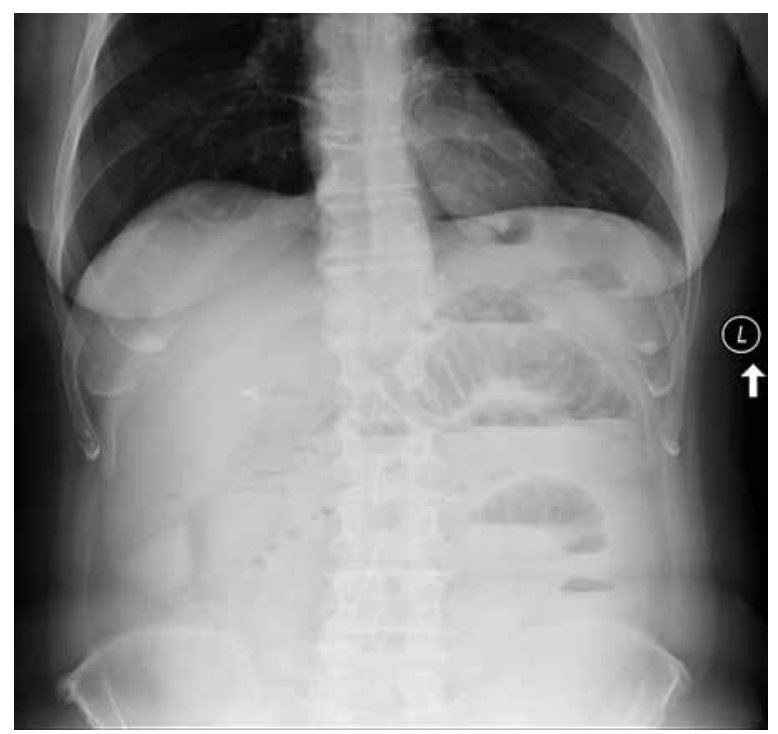

Figure 3. Plain radiograph of the abdomen shows multiple air-fluid levels

The most effective method for treatment is a radical surgical resection of the intestine within surgical margins and regional lymph node assessment (minimum 8 ) [1, 2, 4, 5, 8, 9]. Prognosis depends on locoregional disease and the tumour resectability stage. Poor prognosis factors include: duodenal location, male gender, black ethnicity, older age, poor differentiation, positive margins and lymph node invasion [1]. Systemic treatment in metastatic SBA is based on adjuvant chemotherapy with the combination of fluoropyrimidine (i.e. 5-fluorouracyl or capecytabine) and oxaliplatin as supported by promising outcomes found recently in a few retrospective clinical studies $[1,10]$. It transpires that the protocol used for treating colorectal adenocarcinoma has shown a survival benefit for SBA patients. Of the four prospective clinical trials reported, three studies combining fluoropyrimidine with oxaliplatin have shown similar performance with response rates (RR) of $42 \%$ to $50 \%$ and a median time to progression (TTP), ranging from 7.8 to 9.8 months $[1,11-13]$.
Despite the small bowel possessing the greatest absorption surface area of the alimentary tract, SBA has a 50-fold lower incidence than large bowel adenocarcinoma. There are several hypotheses proposed to explain the apparent resistance of the small intestine to carcinogenesis. It is believed, that rapid turnover of small intestine epithelium eliminates the accumulation of genetic damage and the increased lymphoid tissue of the small intestine provides increased mucosal immune surveillance. Due to rapid transit time of digesta, the concentration of aerophilic Grampositive bacteria increases in the distal ileum and a dilute alkaline environment and lack of bacterial degradation activity decrease the exposure to dietary carcinogens $[1,5]$.

Unfortunately, SBA has a worse prognosis and treatment outcomes compared to colorectal adenocarcinoma. Because access is difficult for any impeding examination as well as the insidious course of the disease, the non-specific symptoms and low prevalence, these factors contribute to there being $32 \%$ of patients with SBA seen at the disease stage IV at the time of diagnosis. Duodenal SBA appears to have a worse prognosis than jejunal or ileal SBA but the reason is unexplained. It may be assumed that incomplete lymph node resection at surgery and misclassifying some patients suffering from adenocarcinoma of the ampulla of Vater as having duodenal SBA could explain this finding; ampullary cancer should be treated with gemcitabine-based regimens $[10,14]$.

A number of ongoing clinical trials are focused on exploring molecular similarities between SBA and colorectal adenocarcinoma in order to find alternative therapeutic methods. A new chance for treatment is provided by targeted therapy using human monoclonal antibodies (cetuximab, bewacizumab), that possess high affinity and specificity for the epidermal growth factor receptor (EGFR). A handful of case reports have also described responses to anti-EGFR therapy in SBA patients with KRAS wild-type tumours $[1,5,15]$. The SBA treatment protocol is nevertheless still vague, mainly due to the low prevalence of SBA and too few prospective clinical trials [10]. 


\section{Conclusions}

Given the rarity of this cancer and a lack of a representative group of SBA patients, we are still far from creating an optimal method for diagnostics and treatment.

Examination of rare tumours is a great scientific challenge and it demands multicentered clinical trials and close collaboration between medical doctors and a wide group of scientists. Elucidating precisely the molecular biology of SBA may not only improve our oncological vigilance, but also contribute to discovery of novel therapeutic agents for those patients specifically at high risk of SBA (i.e. patients with coeliac disease, Crohn's disease and Lynch II syndrome). All treatment decisions should be made on a multidisciplinary basis. Such a group should consist of: an oncological surgeon, clinical oncologist, radiotherapeutist, and specialists in radiology and pathomorphology.

\section{Conflict of interest: none declared}

\section{Joanna Polczyk, MD}

Municipal Hospital

Department of General Surgery

Zamkowa 4, 41-803 Zabrze

e-mail:joannapolczyk@gmail.com

Received: 25 Nov 2016

Accepted: 6 Feb 2017

\section{References}

1. Overman MJ. Rare butreal:Management of small bowel adenocarcinoma. ASCO educational book 2013: 189-193 [asco.org/edbook].

2. Herman R, Pałucki J, Potemski P. Nowotwory jelita cienkiego. In: Krzakowski M, Warzocha K (red.). Zalecenia postępowania diagnostyczno-terapeutycznego w nowotworach złośliwych 2013. Gdańsk:Via-Medica, 2013: 135-139.

3. Didkowska J, Wojciechowska U. Nowotwory złośliwe w Polsce w 2013 roku. Warszawa: Krajowy Rejestr Nowotworów, 2015.

4. Li J, Wang Z, Liu N et al. Small bowel adenocarcinoma of the jejunum: a case report and literature review. World J Surg Oncol 2016; 14: 177. doi: 10.1186/s12957-016-0932-3.

5. Aparicio T, Zaanan A, Svrcek M et al. Small bowel adenocarcinoma: Epidemiology, risk factors, diagnosis and treatment. Dig Liver Dis 2014; 46: 97-104.

6. Siegel R, Naishadham D, Jemal A. Cancer statistics, 2012. CA Cancer J Clin 2012; 62: 10-29.

7. Shenoy S. Genetic risks and familial associations of small bowel carcinoma. World J Gastrointest Oncol 2016; 8: 509-519.

8. Herman J, Szmeja J, Borejsza-Wysocki M et al. Rak jelita cienkiego wyzwanie diagnostyczne. Now Lek 2010; 79:317-319.

9. Overman MJ, Hu CY, Wolff RA et al. Prognostic value of lymph node evaluation in small bowel adenocarcinoma: analysis of the surveillance, epidemiology, and end results database. Cancer 2010; 116: 5374-5382.

10. Halfdanarson TR, Grothey A. Establishing a standard of care for small bowel adenocarcinomas: chalenges and lessons learned. Oncologist 2012; 17: 1133-1134.

11. McWilliams RR, Mahoney MR, Marchello BT et al. Pharmacogenetic dosing by UGT1A1 genotype as first-line therapy for advanced small bowel adenocarcinoma: A North Central Cancer Treatment Group (NCCTG) trial. J Clin Oncol 2012; 30 Suppl 4; abstr 314.

12. Xiang XJ, Liu YW, Zhang L et al. A phase II study of modified FOLFOX as first-line chemotherapy in advanced small bowel adenocarcinoma. Anticancer Drugs 2012; 23: 561-566.

13. Overman MJ, Varadhachary GR, Kopetz $S$ et al. Phase Il study of capecitabine and oxaliplatin for advanced adenocarcinoma of the small bowel and ampulla of vater: A single center, open-label, phase 2 study. J Clin Oncol 2009; 27: 2598-2603.

14. Overman MJ, Pozadzides J, Kopetz $S$ et al. Immunophenotype and molecular characterisation of adenocarcinoma of the small intestine. Br J Cancer 2010; 102: 144-150.

15. Santini $D$, Fratto $M E$, Spoto $C$ et al. Cetuximab in a small bowel adenocarcinoma: a new friend? Br J Cancer 2010; 103: 1305; author reply 1306 